\title{
COROA TOTAL EM RESINA COMPOSTA DIRETA: RELATO DE DOIS CASOS CLÍNICOS
}

\section{DIRECT COMPOSITE RESIN TOTAL CROWN: TWO CASE REPORTS}

\author{
Patric Vinicius Machado De Bitencourt* \\ Simone Ayang De Morais Gandolfi* \\ Helena Alvez da Silva*" \\ Dalila Meazza Damo**
}

Guilherme Anziliero Arossi"*

\begin{abstract}
RESUMO
Dentes com grande destruição coronária submetidos a tratamento endodôntico devem ser reconstruídos por meio da inserção de um pino intrarradicular, com finalidade de se obter retenção para o material restaurador e melhor distribuição de forças sobre o remanescente dental. A reconstrução coronária desses elementos dentais pode ser realizada com técnicas restauradoras indiretas ou diretas. A evolução das técnicas operatórias e das propriedades físicas, mecânicas e estéticas das resinas compostas tem ampliado suas indicações clínicas, e, quando associadas a pinos intracanais cimentáveis, têm permitido avanços nas reconstruções dentárias. Muitas situações clínicas, que tradicionalmente apresentavam soluções protéticas, hoje podem ser resolvidas através de restaurações diretas com resina composta. Este trabalho apresenta dois casos clínicos onde se realizaram alternativas viáveis de reabilitação de dentes com grande destruição coronária utilizando reconstruções totais diretas com uma resina composta micro-híbrida como material de eleição, associada à utilização de um pino de fibra de vidro e um pino metálico pré-fabricado. Em ambos os casos, foram realizadas as cimentações dos pinos intracanais com cimento ionomérico modificado por resina, seguindo o protocolo tradicional para desobturação dos canais radiculares. Associada ao pino de fibra de vidro foi utilizada a técnica da matriz de silicona como meio para reconstruir a coroa dental, enquanto que junto ao pino metálico lançou-se mão da matriz de Ableh. Concluiu-se que as técnicas descritas constituem procedimentos alternativos, de baixo custo e rápida elaboração, conseguindo suprir as necessidades dos pacientes, demostrando ser uma boa opção para reabilitação de grandes destruições coronárias.

DESCRITORES: Técnica para retentor intrarradicular • Dente não vital • Resinas compostas • Coroas • Reconstrução
\end{abstract}

\begin{abstract}
Endodontic treated teeth with large coronal destruction need be restored by inserting an intracanalpost in order to obtain retention for the restorative material and a better mastigatory stress distribution along the tooth. Subsequently, dental techniques of direct or indirect restorations may be used. The constant evolution of the physical, mechanical and aesthetic properties of composite resins has expanded its clinical indications and adhesive cemented posts have enabled advances in dental reconstructions. Many clinical situations, which in the recent past had only prosthetic solution, can now be build-up with composite resin direct restorations. This paper presents two case reports where were performed viable alternatives for restoring teeth with major coronal destruction through direct total crown reconstruction, with a micro-hybrid composite resin associated with glass fiber post and prefabricated metal post. Both cases were carried out with adhesive cementation of the post, using a resin modified glass ionomer cement. Associated with the glass fiber post, a silicona matrix technique was used as a mean to reconstruct the tooth crown, while with the metallic post it was used the Ableh matrix. It was concluded that techniques described in this work are alternative low cost and fast procedures, which could meet the needs of patients, being a good option for rehabilitation of dental coronary destruction.
\end{abstract} DESCRIPTORS: Post and core technique • Tooth, nonvital • Composite resins • Crowns • Reconstruction

\footnotetext{
* Cirurgiões-dentistas graduados pelo Curso de Odontologia (ULBRA Torres/RS) - Responsáveis pela parte clínica do caso.

** Graduandas do Curso de Odontologia (ULBRA Torres/RS) - Responsáveis pela escrita do artigo.

*** Doutor em Genética e Toxicologia Aplicada; Professor do Curso de Odontologia e do Programa de Pós-Graduação em Promoção da Saúde da ULBRA Torres/RS - Professor orientador. E-mail: guilhermeclinica@gmail.com
} 
DE BITENCOURT

GANDOLFI SAN

SILVA HA

DAMO DM

AROSSI GA

COROA TOTAL EM

RESINA COMPOSTA

DIRETA: RELATO

DE DOIS CASOS

CLÍNICOS

66

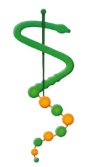

REV, ODONTOL.

UNIV, CID, SÃO

PAULO

$2016 ; 28(1): 65-$

77, JAN-ABR

\section{IN T RO DUÇ ÃO}

Alterações orais podem gerar mudanças no comportamento do indivíduo, conduzindo à insatisfação com a aparência e prejuízo na aceitação social. É possível relacionar a perda dentária com a qualidade de vida, através de instrumentos que mensuram a OHRQoL - Oral Health Related Quality of Life. Esse é um conceito multidimensional, que inclui a avaliação subjetiva da saúde oral do indivíduo, aspectos funcionais, expectativas e satisfação; além de ser reconhecido pela Organização Mundial de Saúde (OMS) por possuir instrumentos validados e ter grandes aplicações em pesquisa ${ }^{1}$. Estudos indicam evidências de que a perda dentária está diretamente relacionada com menor OHRQoL, influenciando nos aspectos físicos, psicológicos e sociais do indivíduo ${ }^{2,3}$, ${ }^{4}$. A reabilitação de dentes tratados endodonticamente gera discussões entre clínicos e pesquisadores e caracteriza-se pela falta de um protocolo clínico padronizado, universalmente aceito como o melhor. Existem várias formas de se realizar a mesma reabilitação: utilizando pinos pré-fabricados, metálicos fundidos ou até mesmo restaurações diretas sem a presença de pinos intracanais, dependendo da perda coronária - cada uma com suas vantagens e desvantagens ${ }^{5}$. Tais dentes merecem um cuidado especial devido à sua menor resistência mecânica quando comparados a dentes com vitalidade pulpar, devido à perda de estrutura dentária, principalmente de dentina, decorrente de fraturas coronárias, lesões cariosas, erosão, abfração. Por isso, é importante preservar o máximo de tecido sadio para manter a integridade da estrutura ${ }^{6}$. Soma-se a isso a perda da vitalidade pulpar, que diminui a umidade dentinária gerando, com isso, prejuízo da capacidade de deformação elástica do elemento dental, tornando-o mais friável ${ }^{7}$.

O tipo de restauração a ser confeccionada em dentes tratados endodonticamente dependerá do grau de destruição da coroa, do dente envolvido, do suporte ósseo, do tipo de prótese e dos tipos de forças às quais esses dentes serão submetidos. A inter-relação desses fatores pode indicar o uso de uma retenção intracanal que proporcione retenção e resistência ao elemento dental a ser reconstruído ${ }^{8}$. Em casos em que só estiver presente a raiz, os pinos representam o único meio disponível para se confeccionar as coroas e devolver ao paciente o dente com forma e função ${ }^{9}$. A seleção correta do pino é determinante para o sucesso do procedimento restaurador. Nessa escolha, devem ser considerados fatores relacionados tanto ao elemento que será restaurado - oclusão, posição de dente no arco, remanescente dentário, configuração e diâmetro do canal - quanto às características dos pinos - comprimento, diâmetro, formato, configuração superficial e composição do pino $^{10}$. Pinos diretos são preferencialmente indicados em dentes que irão receber restaurações unitárias, já os indiretos em dentes que serão pilares de prótese fixa ${ }^{11}$.

O comprimento do pino está diretamente relacionado com a retenção e deve satisfazer tanto às exigências funcionais quanto às biológicas, tendo como regras (1) pelo menos 3 a $4 \mathrm{~mm}$ de material obturador endodôntico devem permanecer na região apical; (2) uma relação de 1:1 entre a altura da coroa e o comprimento radicular do pino deve ser respeitada; (3) o pino deve se estender ao menos por metade do comprimento da raiz suportada por tecido ósseo; (4) as paredes circundantes do canal (dentina) devem ser desgastadas ao mínimo durante o preparo para a colocação do pino, para não fragilizar ainda mais o dente ${ }^{12}$.

A cimentação dos pinos pré fabricados pode ser realizada com cimento de ionômero de vidro ou cimento resinoso, preferencialmente sistemas duais ou quimicamente ativados, devido à dificuldade de acesso com luz do aparelho fotopolimerizador na região radicular ${ }^{13}$. A indicação de uma técnica de cimentação adesiva tem possibilitado um aumento significativo na capacidade de retenção dos pinos intrarradiculares, além de propiciar um reforço da estrutura dentária ${ }^{14,15}$.

A evolução das resinas compostas tem sido comprovada na melhoria do seu comportamento estético e no aumento da sua resistência à compressão e à abrasão e, aliadas aos sistemas adesivos, têm sido muito empregadas para restaurações de 
dentes anteriores e posteriores ${ }^{11,16,17}$. Em função disso, é possível indicar restaurações extensas de resina composta em dentes anteriores, tendo como vantagem a facilidade na técnica, que dispensa a etapa laboratorial. Além disso, as resinas fornecem uma grande diversidade de cores e efeitos, e os consertos que se tornarem necessários são facilmente contornados sem despesas laboratoriais, com total controle do cirurgião-dentista ${ }^{5,17,18}$.

Sabe-se que a cerâmica é um material com ótimas propriedades funcionais e estéticas e longevidade comprovada, porém, apresenta desvantagens como maior tempo clínico necessário a sua confecção, além de elevado custo para o paciente e profissional em relação às restaurações diretas $^{19}$. Por esse motivo, aumentou o interesse no desenvolvimento de estudos que mostrem a possibilidade de uso de materiais adesivos de forma direta nas situações de grandes destruições coronárias, o que pode favorecer uma grande parte da população que não dispõe dos recursos necessários para realizar o procedimento indireto. Este trabalho tem como objetivo apresentar o uso de resinas compostas na reconstrução de dentes amplamente destruídos por meio de dois relatos de casos clínicos.

\section{RELATO DE CASO CLÍNICO 1}

A realização deste caso foi avaliada e aprovada pelo Comitê de Ética e Pesquisas em Seres Humanos da Universidade Luterana do Brasil, protocolado no número CEP-ULBRA 2011-181H.

Paciente S.S., vigilante, com 32 anos, foi encaminhado à clínica do Projeto de Atendimento Odontológico para Núcleos Especiais ULBRA Torres, apresentando como queixa principal seu incisivo lateral inferior direito - dente 42 - com grande perda de estrutura coronária, interferindo na estética e função (Figura 1).

Na primeira consulta, após exame clínico e verificação radiográfica da qualidade da obturação endodôntica, optou-se por um tratamento reabilitador utilizando-se um retentor intrarradicular estético ${ }^{20}$ associado à restauração direta de resina composta. Foram produzidos modelos de estudo do paciente e, na fase laboratorial, enceramento diagnóstico e realização de moldagem desse enceramento com a pasta pesada da silicona de condensação Speedex (Vigodent, Rio de Janeiro, RJ, Brasil). A seguir, procedeu-se ao recorte da moldagem, obtendo-se a guia de silicona para inserção de resina composta. Na segunda sessão, calculou-se a quantidade de material obturador a ser

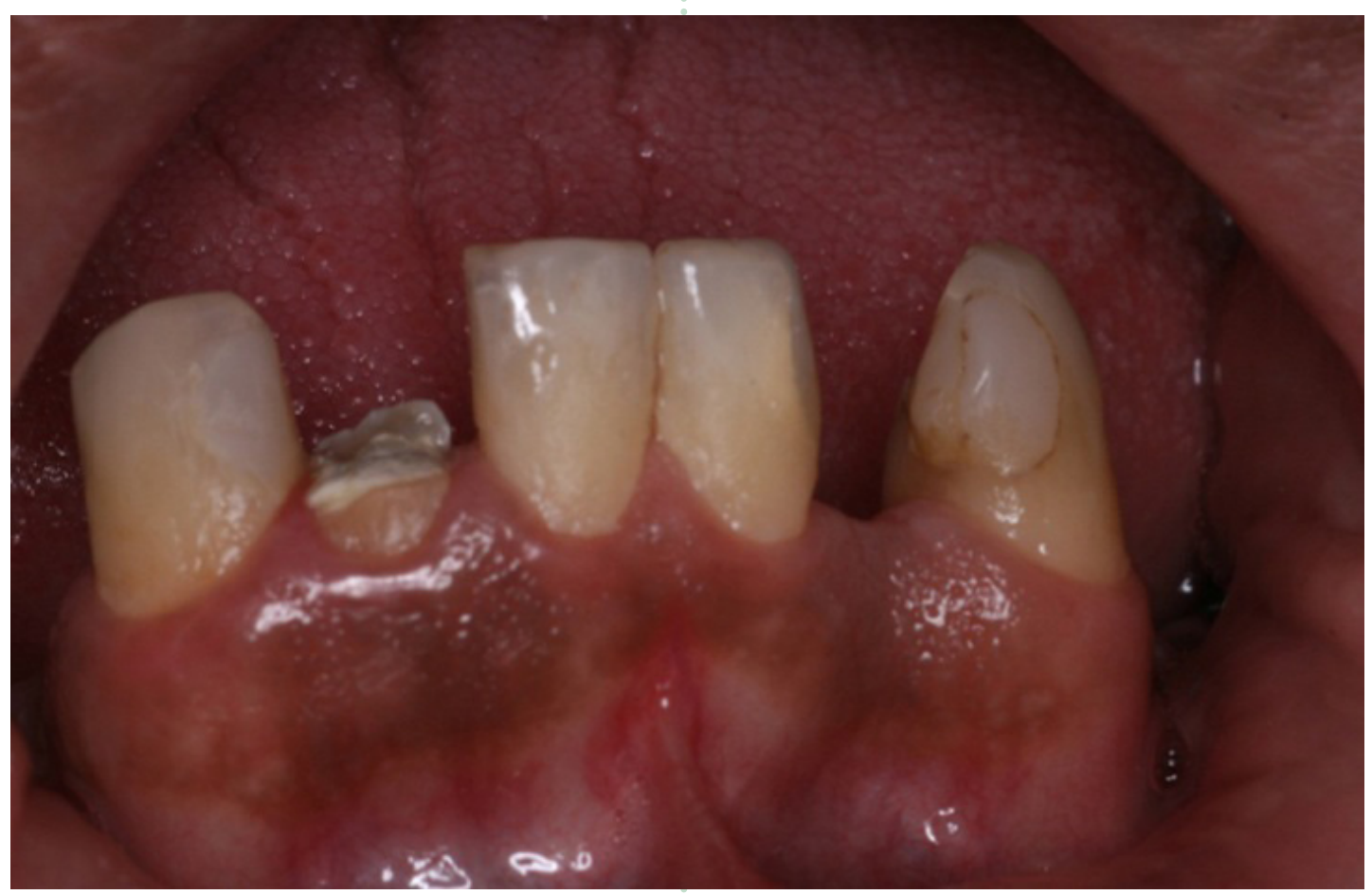

Figura 1 - Foto inicial, grande perda de estrutura coronária.
DE BITENCOURT $P V M$

GANDOLFI SAN

SILVA HA

DAMO DM

AROSSI GA

COROA TOTAL EM RESINA COMPOSTA

DIRETA: RELATO

DE DOIS CASOS

CLÍNICOS

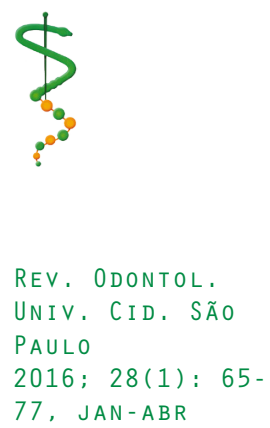


DE BITENCOURT

$P V M$

GANDOLFI SAN

SILVA HA

DAMO DM

AROSSI GA

COROA TOTAL EM RESINA COMPOSTA

DIRETA: RELATO

DE DOIS CASOS CLÍNICOS

$\therefore 68$ removido $(10 \mathrm{~mm})$, de forma que $4 \mathrm{~mm}$ de guta-percha ficaram intactos para garantir o selamento apical. Iniciou-se a desobturação, confirmando-se o comprimento da broca Largo (Microdent, São Paulo, SP, Brasil), com régua endodôntica milimetrada e cursor de borracha. Após o preparo do canal radicular ${ }^{12}$, este foi limpo com auxílio de lima endodôntica tipo K \#40 (Dentsply-Maileffer, Rio de Janeiro, RJ,
Brasil) e algodão embebido em álcool e seco com cones de papel absorvente, com intuito de retirar algum resquício de material obturador contendo eugenol ${ }^{21}$. Logo, prosseguiu-se à marcação e prova do pino Reforpost número 1 (Angelus, Londrina, PR, Brasil) no comprimento de desobturação.

Para a cimentação do pino foi selecionado o cimento de ionômero de vidro mo-

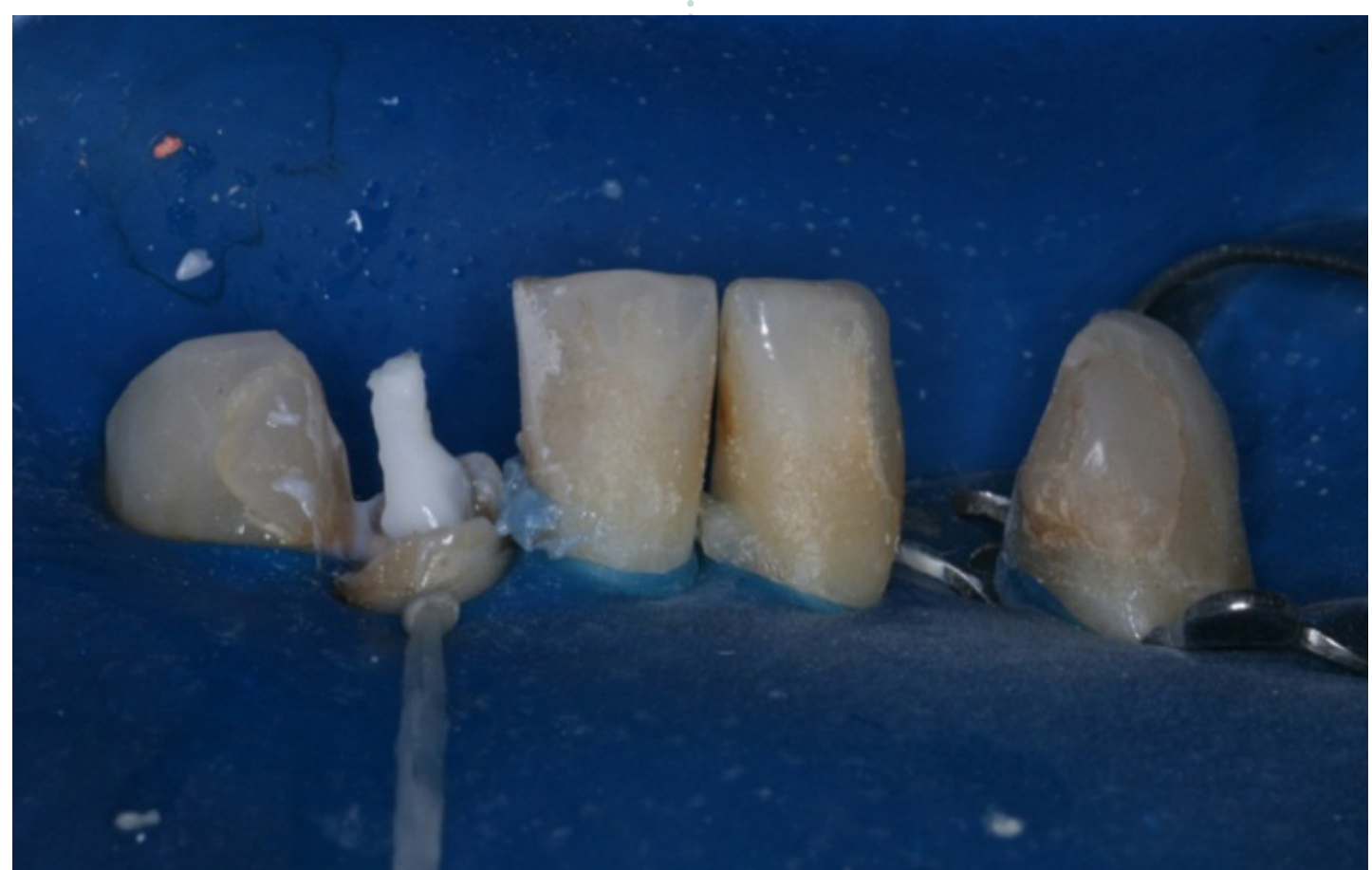

Figura 2 - Pino inserido no canal

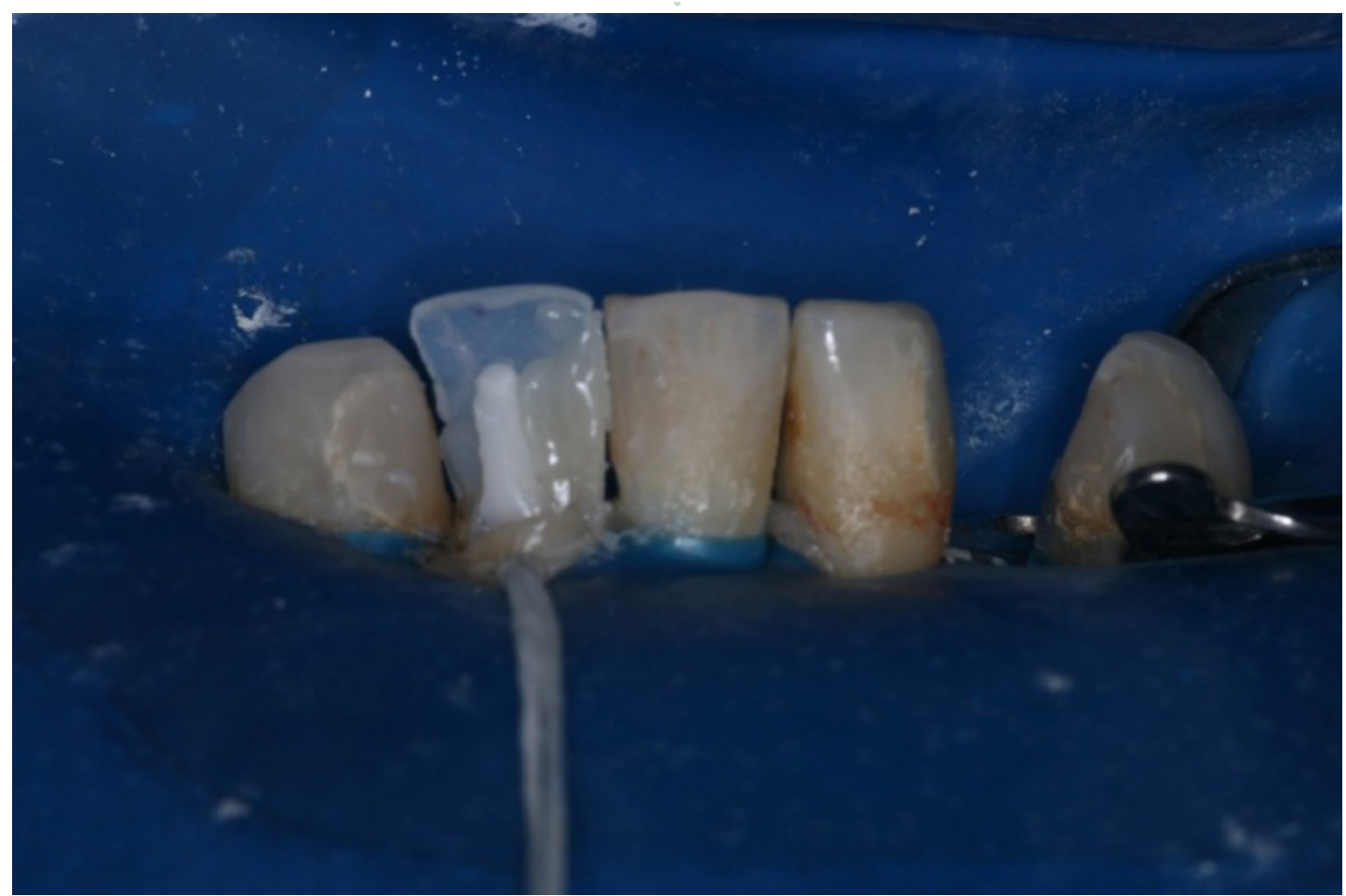

REV, ODONTOL

UNIV, C I D , SÃO PAULO 


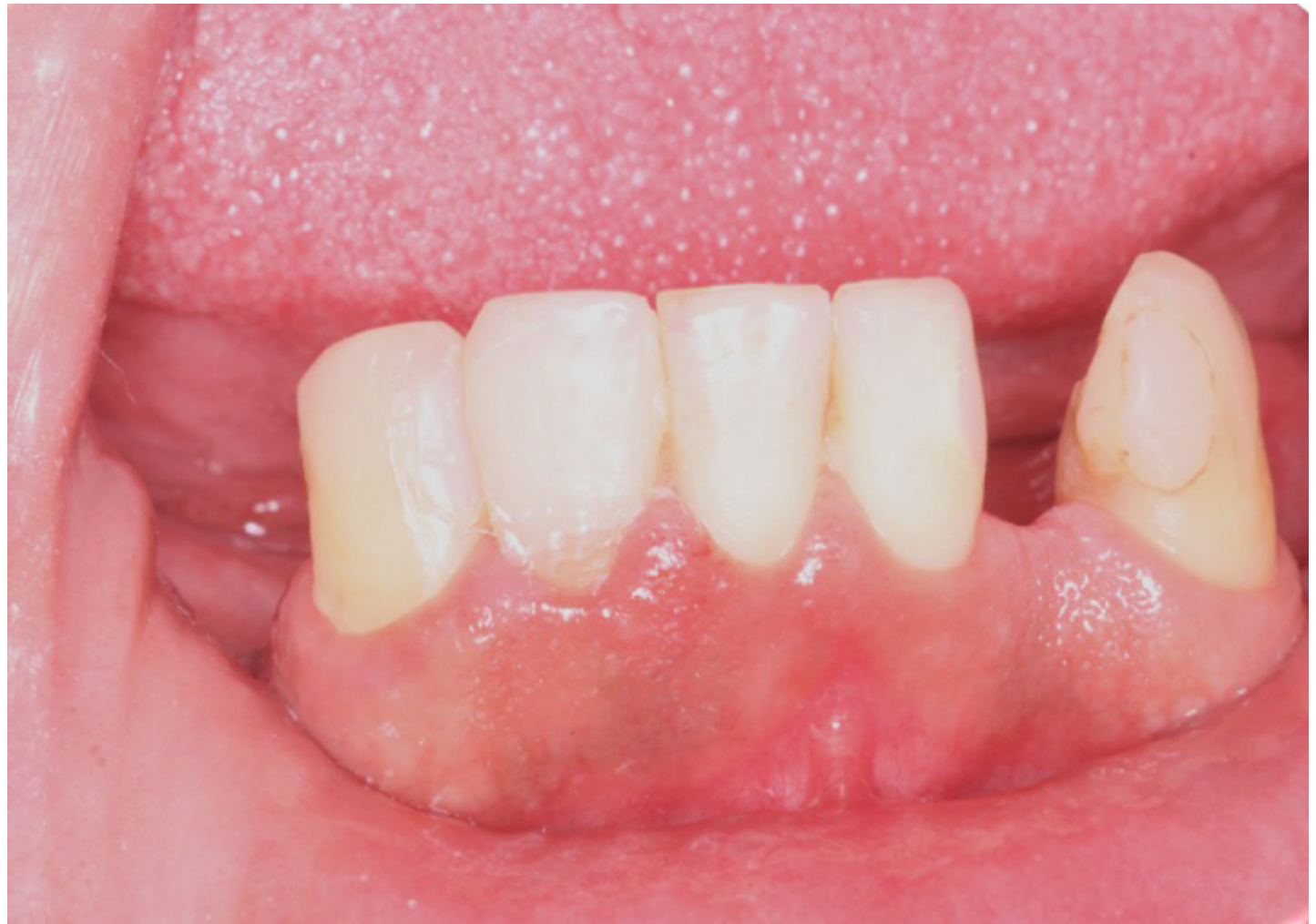

Figura 4 - Foto após acabamento e polimento.

dificado por resina RelyX ${ }^{\mathrm{TM}}$ Luting 2 (3M/ ESPE, São Paulo, SP, Brasil), manipulado e inserido no canal radicular com auxílio de broca Lentulo número 35 (Dentsply-Maileffer, Rio de Janeiro, RJ, Brasil). Com o canal devidamente preenchido, o cimento foi aplicado à superfície do pino previamente limpo com álcool e embebido em adesivo Single Bond 2 devidamente fotoativado (3M/ESPE, São Paulo, SP, Brasil). O pino de fibra foi, então, inserido no canal e o extravasamento de cimento retirado, seguido de fotopolimerização e do corte do excesso de pino em alta rotação com ponta diamantada esférica 1014 (KG Sorensen, SP, Brasil) (Figura 2) ${ }^{20}$.

Com o pino devidamente cimentado mediante isolamento absoluto do campo operatório, os dentes adjacentes foram protegidos para realização de protocolo adesivo - condicionamento com ácido fosfórico $37 \%$ por 30 segundos e aplicação de adesivo seguido de fotopolimerização por 30 segundos ${ }^{12}$. Na reconstrução dental em resina composta, a guia de silicona obtida foi empregada para inserir uma fina camada resina composta Charisma (Heraeus-Kulzer, Hanau, Alemanha) cor Incisal na superfície lingual da coroa em reconstrução (Figura 3).

A partir daí, deu-se início à inserção de resina Opallis (FGM, Joinville, SC, Brasil) cor A2 para dentina no corpo da restauração, reproduzindo os mamelões dentinários. Utilizou-se resina Opallis cor A2 para esmalte nos bordos e face vestibular da coroa, sempre em pequenos incrementos, fotoativados individualmente, utilizando-se fotopolimerizador Optilight LD III (Gnatus, Ribeirão Preto, SP, Brasil) com uma potência de $1200 \mathrm{~mW} / \mathrm{cm}$, para garantir um máximo de desempenho clínico do material ${ }^{12} \mathrm{e}$, dessa forma, finalizou-se a construção da coroa total em resina composta. Após sete dias, o paciente retornou ao atendimento, quando foram feitas manobras de acabamento e polimento da restauração ${ }^{22}$. Para tal, foram utilizados ponta diamantada $n^{\circ} 2135 \mathrm{~F}$ (KG Sorensen, São Paulo, SP, Brasil), discos tipo sof-lex (3M/ESPE, Nove Veneza, Sumaré, SP) em três granulações, e, por fim, ponta de polimento Pogo Disco (Dentsply, Petrópolis, RJ, Brasil) até a superfície apresentar-se perfeitamente lisa e brilhante. (Figura 4).
PVM

GANDOLFI SAN

SILVA HA

DAMO DM

AROSSI GA

COROA TOTAL EM RESINA COMPOSTA

DIRETA: RELATO

DE DOIS cASOS

CLÍNICOS
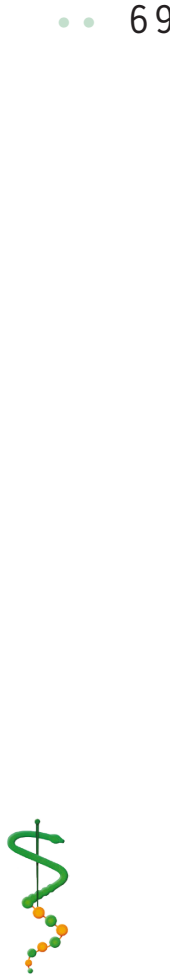

REV. ODONTOL. Univ. Cid. São PAULO

2016; 28(1): 6577 , JAN-ABR 


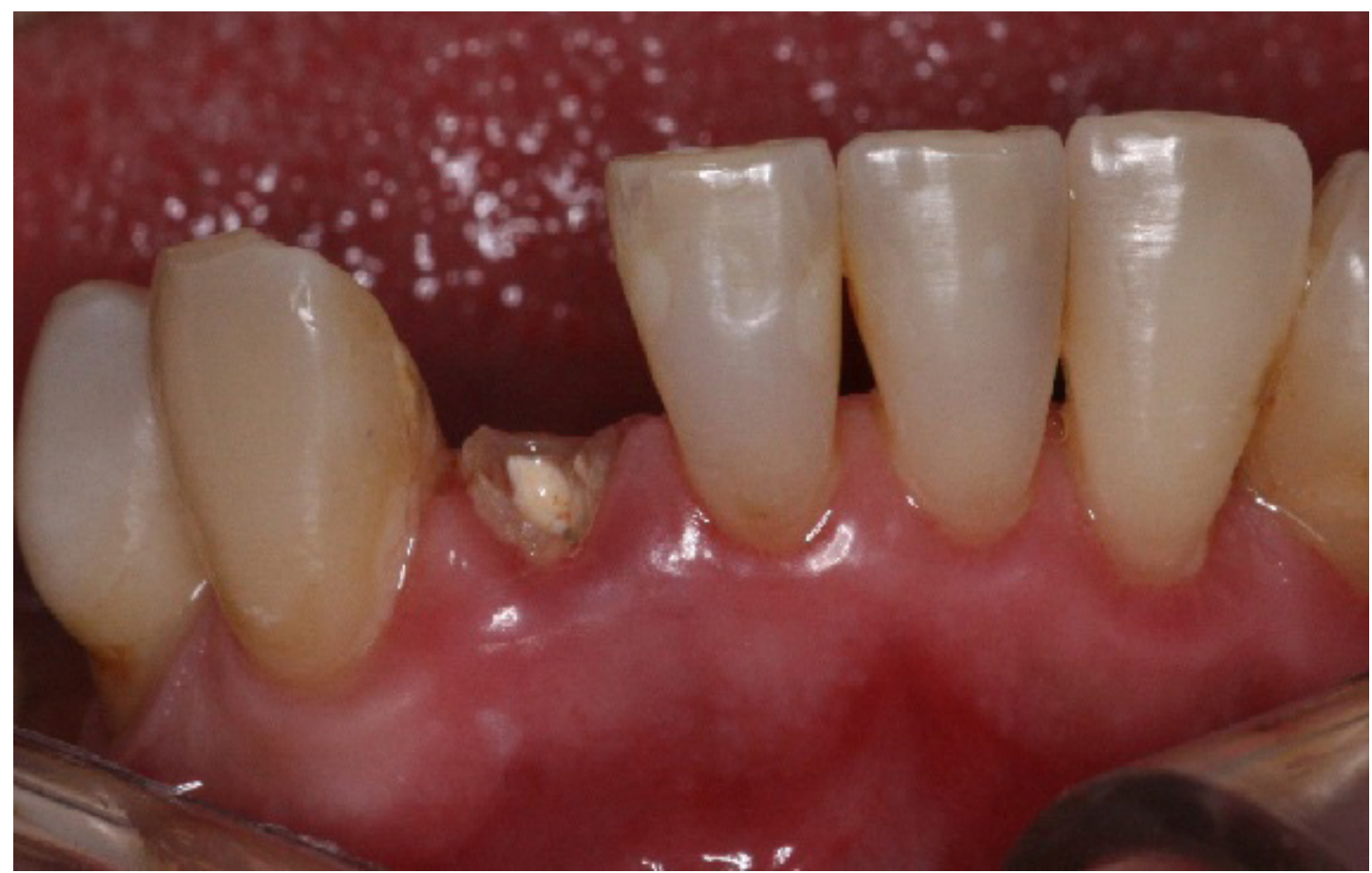

Figura 5 - Foto inicial, extensa destruição coronária.

Este caso foi avaliado e aprovado pelo Comitê de Ética em Pesquisa em Seres Humanos da ULBRA sob o protocolo CEP-ULBRA 2011-088H. Paciente M.S.S.S., 44 anos, sexo feminino, apresentou-se na clínica do Projeto de Atendimento Odontológico para Núcleo Especiais e Sala de Espera para reabilitação do elemento 42. Esse dente apresentava destruição total da coroa dental e tratamento endodôntico satisfatório comprovado clínica e radiograficamente, sem dor ou qualquer tipo de sintomatologia. (Figura 5).

O tratamento escolhido foi a instalação de um pino intracanal pré-fabricado metálico $^{14}$ e restauração com resina composta direta. Iniciou-se com a remoção do pino e da coroa provisória que se encontrava frouxa e desadaptada. Após a mensuração radiográfica do comprimento total da obturação endodôntica, procedeu-se à desobturação parcial do canal com broca Largo no 2 (Microdent, São Paulo, SP, Brasil) removendo-se 2/3 do material obturador - aproximadamente $10 \mathrm{~mm}$. Uma vez concluída a desobturação parcial e confirmação com uma sonda milimetrada (Neumar, Caieiras, SP, Brasil), foi realizada uma limpeza do canal com algodão embebido em álcool a 95º, junto a uma lima tipo K \#40 (Maillefer/Dentsply, Petró- polis, RJ, Brasil), com o objetivo de eliminar produtos orgânicos e contaminações derivados do material de obturação endodôntica e da técnica operatória ${ }^{20}$. Após essas etapas, foi escolhido o pino metálico pré-fabricado (Angelus, Londrina, PR, Brasil) e introduzido no canal para análise clínica e radiográfica de sua adaptação.

Na cimentação adesiva foi utilizado o cimento de ionômero de vidro modificado por resina Rely $\mathrm{X}^{\mathrm{TM}}$ Luting 2 (3M/ ESPE, St. Paul, Minessota U.S.A.) (Figura 6).

Com a broca Lentulo (Maillefer, Ballaigues, Suíça), foi introduzido o cimento no interior do conduto, seguido da inserção e adaptação do pino e polimerização por 60 segundos com aparelho Optilight LD III (GNATUS, Ribeirão Preto, SP, Brasil) com uma potência de $1200 \mathrm{~mW} / \mathrm{cm}$. Após recobrimento de todo o pino com o mesmo material cimentador, foi confeccionado um núcleo de preenchimento em resina composta Charisma OA3 (Heraeus-Kulzer, Hanau, Alemanha).

Foi então realizado o preparo para coroa total com ponta diamantada tronco cônica n ${ }^{\circ} 2135$ (KG SORENSEN, Cotia, SP, Brasil), de forma a se obter aproximadamente $2 \mathrm{~mm}$ de espessura em toda a extensão do preparo, o que é essencial para que a resina composta desenvolva estru- 


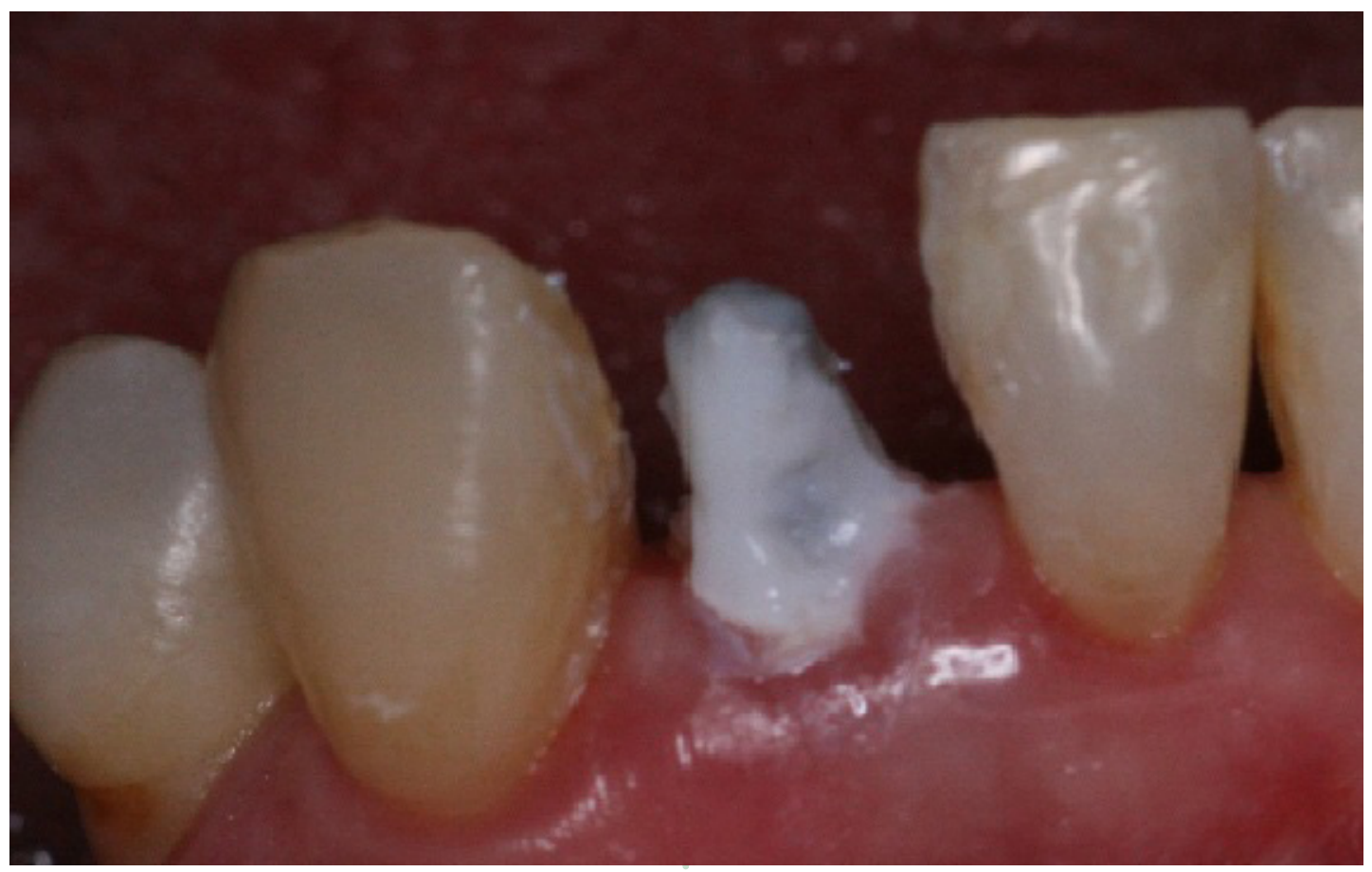

DE BITENCOURT $P V M$

GANDOLFI SAN

SILVA HA

DAMO DM

AROSSI GA

COROA TOTAL EM RESINA COMPOSTA

DIRETA: RELATO

DE DOIS CASOS

CLÍNICOS

Figura 6 - Cimentação do pino

tura suficiente para resistir aos esforços da função mastigatória ${ }^{23}$. Na sequência, com o isolamento absoluto, procedeu-se ao condicionamento com ácido fosfórico a 37\% (Villevie, Joinville, SC, Brasil), e à aplicação do adesivo Single Bond 2 (3M ESPE, St. Paul, Minessota U.S.A.), permitindo o início do procedimento de acomodação da resina composta.

Optou-se pela utilização das matrizes pré-fabricadas para dentes anteriores TDV -coroa oca de poliacetato tipo Ableh (TDV, Pomerode, SC, Brasil) - compatível com o tamanho da $\operatorname{coroa}^{20}$. A resi-

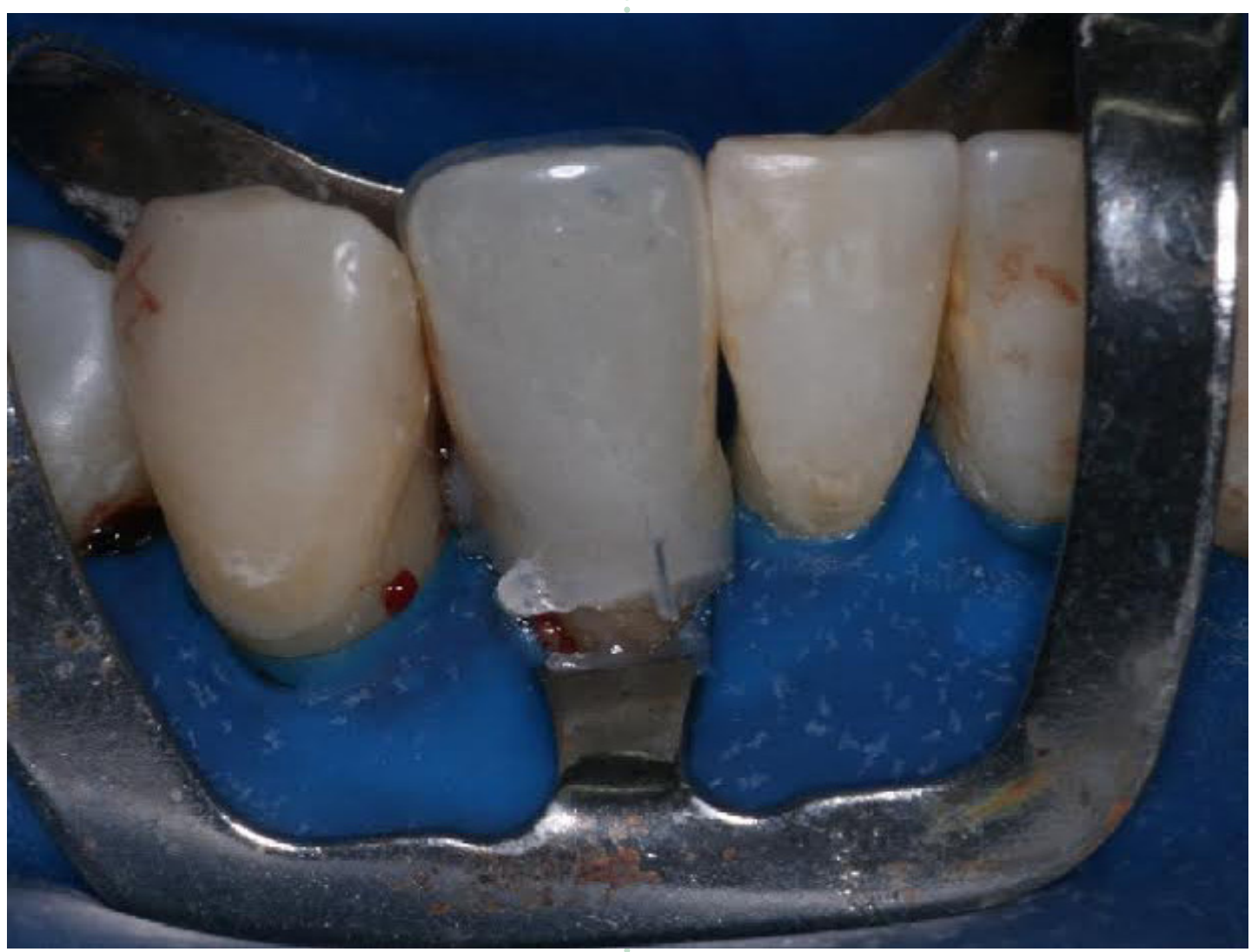

Figura 7 - Preenchimento e adaptação da matriz

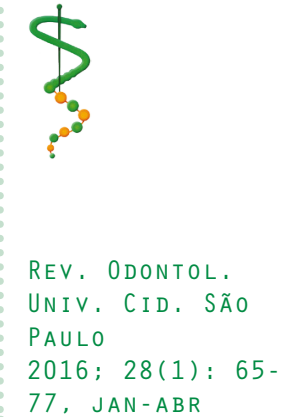




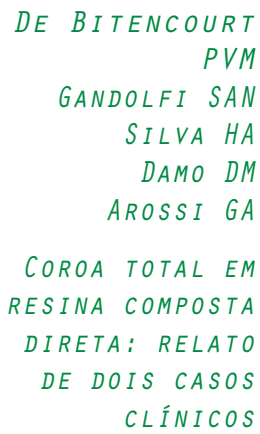

REV, ODONTOL

UNIV, C I D , S ÃO

PAULO

$2016 ; 28(1): 65$

$77, \mathrm{JAN}-\mathrm{ABR}$

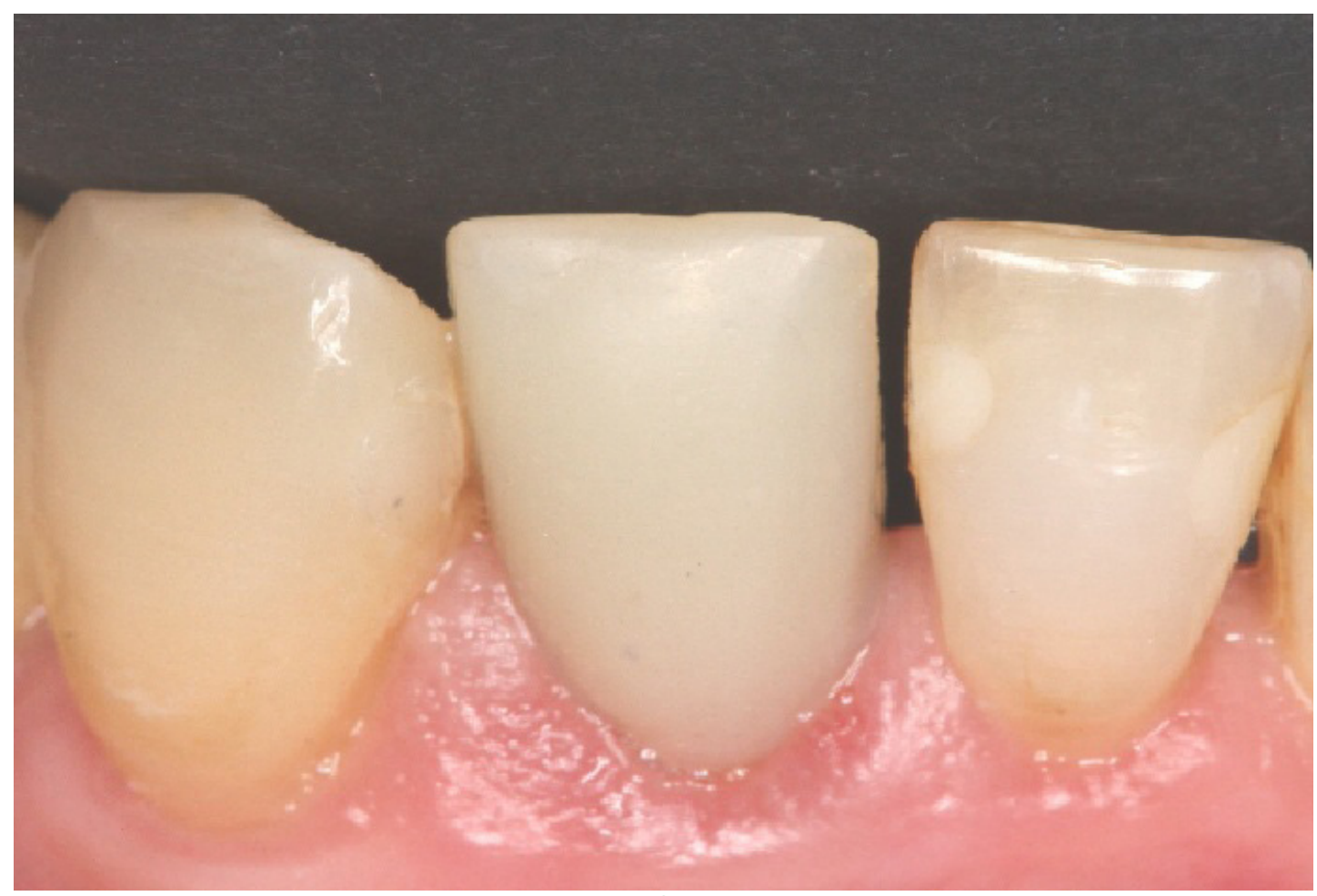

Figura 8 - Aspecto após 5 meses

na composta escolhida foi micro-híbrida Charisma OA3 (Heraeus-Kulzer, Hanau, Alemanha). Após a individualização e adaptação da matriz, com recortes feitos com tesoura, a fim de se adequar a altura da matriz aos dentes adjacentes da paciente, procedeu-se ao preenchimento da mesma com a resina selecionada. Uma vez preenchida, a matriz foi adaptada ao preparo dental e, então, foi feita a remoção dos excessos de material extravasado com sonda exploradora (Figura 7).

A polimerização foi realizada por longo período, aproximadamente 60 segundos em cada face do dente, a fim de garantir a adequada cura do material. Removeu-se a matriz com lâmina de bisturi $N^{0} 11$ (Life, Recife, $P E$, Brasil) e com a utilização de ponta diamantada $n^{\circ}$ 2135FF (KG SORENSEN, Cotia, SP, Brasil), em alta rotação, com refrigeração, foram removidos os excessos de resina composta.

Realizou-se o ajuste oclusal com pontas diamantadas e discos sequenciais de acabamento e polimento. O polimento final com discos de contorno e polimento Sof-Lex pop-on (3M ESPE, St. Paul, U.S.A.) foi realizado na consulta seguinte, utilizando-se os discos em baixa rotação da maior granulação para a menor granulação até a obtenção da lisura superficial.
Observa-se o aspecto do procedimento restaurador após 5 meses na Figura 8.

\section{I SCUSSÃO}

Apesar da evolução dos materiais restauradores, a estrutura dental sadia continua sendo insubstituível. Por isso, em todas as técnicas restauradoras, inclusive em dentes extensamente destruídos, procura-se respeitar ao máximo o remanescente de estrutura dental. A durabilidade e o sucesso do tratamento reabilitador dependerão mais de como o paciente vai cuidar dessa nova situação dentária na qual ele se encontra do que do desempenho do material propriamente dito ${ }^{24}$. Devido à grande perda coronária apresentada em ambos os casos, o tratamento mais comumente indicado seria o núcleo metálico fundido associado à coroa metalocerâmica ${ }^{25}$.

Pinos pré-fabricados são indicados como alternativa viável ao núcleo metálico fundido para retenção intrarradicular, pois utilizam uma técnica de inserção relativamente simples, consomem menor tempo clínico ${ }^{26,27}$, podendo serem confeccionados em sessão única. Além disso, em combinação com cimentações adesivas, esses pinos apresentam características biomecânicas que se assemelham à estrutura dentinária, caracterizando biomimetismo 
e favorecendo a distribuição das tensões à estrutura radicular. Dessa maneira, minimizam-se os riscos de fratura radicular em relação à utilização de núcleos metálicos fundidos ${ }^{14}$.

Atualmente, pinos de fibra de vidro têm grande aceitação pela resistência, flexibilidade e cor, favorecendo a estética. Proporcionam, também, facilidade de manuseio e de remoção se necessária, compatibilidade química com materiais resinosos e custo favorável ${ }^{28}$. Um pino metálico pré-fabricado tipo rosqueável também pode ser utilizado como alternativa de retentor intrarradicular. Esse tipo de pino tem por características instalação rápida, fácil, de baixo custo, dispensando as etapas de moldagem e laboratorial. Assim, os pinos metálicos pré-fabricados permitem preparos mais conservadores, estão disponíveis em várias formas e tamanhos, e estão indicados para casos com perda da estrutura dental igual ou superior a 2/3 da coroa $^{29}$. Além disso, o cimento endodôntico à base de eugenol influencia negativamente a adesão, sendo o substrato adesivo outro motivo para não se realizar a inserção do pino imediatamente após a obturação endodôntica ${ }^{30}$. O núcleo metálico fundido e pinos pré-fabricados, associados ou não a pinos acessórios, apresentam valores semelhantes de resistência à tração, quando cimentados com cimento resinoso ${ }^{9,15}$.

A escolha do cimento de ionômero de vidro modificado por resina RelyX ${ }^{\text {TM }}$ Luting 2 (3M/ ESPE, St. Paul, Minessota U.S.A.) ocorreu devido a esse cimento possuir polimerização dual, auto e fotopolimerizável, o que permite uma polimerização completa mesmo sem a penetração da luz nas regiões mais profundas do conduto radicular, tem uma boa aderência e retenção tanto ao pino metálico quanto ao pino de fibra e ao substrato dentário via quelação superficial do cálcio. Por ser modificado por resina, possui uma maior capacidade de absorver tensões geradas pelas forças mastigatórias sobre o pino durante a função, reduzindo, dessa forma, o estresse acumulado nas paredes do remanescente radicular, o que contribui para a prevenção de fraturas radiculares ${ }^{14,31}$. Alguns estudos têm investigado uma possível influência do operador nos resultados de procedimentos adesivos, principalmente com cimentos resinosos, que exigem uma técnica adesiva mais complexa. Esses estudos indicam que a resistência de união do pino à dentina radicular foi significativamente afetada pelo operador, entretanto, para operadores com menos experiência, o cimento resinoso autoadesivo RelyX apresentou estatisticamente o melhor desempenho ${ }^{32}$.

Com a evolução das porcelanas, hoje há a possibilidade de restabelecer coroas unitárias anteriores com restaurações puramente cerâmicas ${ }^{19}$. Somando-se a isso, as resinas compostas vêm sofrendo uma intensa evolução estética e mecânica, sendo capazes de serem indicadas para reabilitar uma coroa total perdida, seja pela técnica direta ou indireta ${ }^{24}$. Enquanto as restaurações cerâmicas indiretas apresentam como vantagem sua durabilidade enquanto material, uma vez que são mais resistentes ao desgaste e ao manchamento do que as resinas compostas diretas ${ }^{33}$, estas são de mais rápida execução e conseguem reabilitar satisfatoriamente, tanto estética como mecanicamente ${ }^{20}$, e com um menor custo ${ }^{34}$.

Do ponto de vista estético, a reconstrução de dentes tratados endodonticamente é um desafio para o cirurgião-dentista, ainda mais quando se trata de paciente com poucos recursos financeiros. Neste estudo, optou-se pela reconstrução direta com resina composta, pois esse método, que exclui a fase laboratorial, já vem sendo utilizado como opção de reduzir custos e alcançar a estética favorável ${ }^{20,24,35}$. A escolha do material restaurador é um fator importante para a longevidade da restauração. A resina utilizada neste estudo vai ao encontro de Arossi et al. ${ }^{20}$ (2010), quando esses autores relataram dois casos clínicos, com uso de pino de fibra de vidro para retenção do material restaurador e utilizaram resina composta micro-híbrida, por possuir grande quantidade de carga, o que aumenta consideravelmente a resistência, podendo ser indicada para amplas reconstruções ${ }^{17}$. O polimento realizado em uma segunda sessão se justifica porque após uma semana a resina já sofreu a expansão higroscópica e finalizou
DE BITENCOURT $P V M$

GANDOLFI SAN

SILVA HA

DAMO DM

AROSSI GA

COROA TOTAL EM

RESINA COMPOSTA

DIRETA: RELATO

DE DOIS cASOS

CLÍNICOS
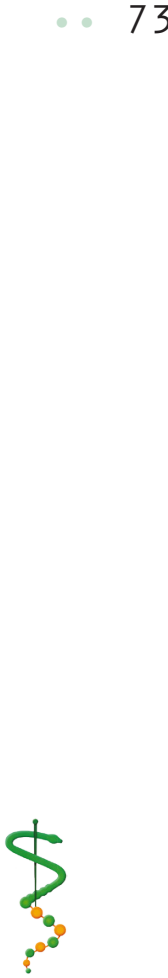

REV, ODONTOL.

UNIV, CID, SÃO

paulo

2016; 28(1): 65 -

77 , JAN-ABR 
DE BITENCOURT

$P V M$

GANDOLFI SAN

SILVA HA

DAMO DM:

AROSSI GA

COROA TOTAL EM

RESINA COMPOSTA

DIRETA: RELATO

DE DOIS CASOS

CLÍNICOS

$74 \ldots$

REV, ODONTOL,

UNIV. CID, SÃO

PAULO

2016; 28(1): 65-

77 , JAN-ABR sua presa, de modo que podemos ajustar corretamente a oclusão sem prejudicar estruturalmente a restauração ${ }^{36}$ Uma das desvantagens da resina composta é a susceptibilidade ao manchamento, porém, Korkut et al.32, em 2013, realizaram acompanhamento por seis meses em três casos clínicos de restaurações em dentes anteriores com resina composta, observando que quando procedimentos de acabamento e polimento são bem conduzidos e o paciente é bem orientado quanto à higienização e dieta, as restaurações diretas de resina composta são um método confiável para chegar ao bom resultado estético.

As técnicas relatadas neste trabalho constituem alternativas viáveis para situações clínicas específicas e, também, como opção para muitos pacientes cuja condição social não permite a confecção de uma restauração indireta ou de porcelana. A praticidade, já que o número de sessões é menor quando comparado às restaurações indiretas, e a efetividade do tratamento, já que o paciente consegue recuperar sua estética e função, também são vantagens que vêm popularizando a técnica tanto entre profissionais quanto pacientes.

Contudo, com o passar do tempo, o compósito perde seu brilho superficial e pode ocorrer manchamento causado por tabaco e/ou alimentos com excesso de corantes, que fazem com que esse trabalho tenha um tempo relativamente curto se o paciente não colaborar com a preservação da restauração. Essa preservação se dá por meio da adesão às orientações do profissional, que incluem dieta parda, evitar grandes variações de temperatura na boca e controlar parafunções tipo bruxis$\mathrm{mo}^{37}$. Uma observação importante é que o sucesso da técnica operatória também depende da habilidade do profissional, pois a restauração é realizada diretamente em boca onde existem desafios que devem ser contornados como controle de umidade, adaptação das margens com o auxílio de matrizes e cunhas, confecção do ponto de contato, o que eleva o tempo clínico necessário ao procedimento ${ }^{38}$.

\section{CONCLUSÃO}

O desempenho clínico do tipo de restauração abordada neste trabalho deve ser considerado dentro de um contexto de recuperação da estética e funcional do paciente como um todo, contribuindo para a adesão deste ao tratamento e servindo como objeto de transformação de atitudes em relação à sua saúde bucal.

As técnicas aqui apresentadas conseguiram suprir as necessidades dos pacientes, concordando com a literatura existente no que diz respeito ao excelente potencial restaurador das resinas compostas, quando criteriosamente empregadas juntamente com um planejamento integral do paciente. Além disso, permitem que se restabeleça função e estética imediatamente, suprindo anseios dos pacientes, muitas vezes melhorando sua qualidade de vida em curto prazo. 
1. Sischo L, Broder HL. Oral Healthrelated Quality of Life: What, Why, How, and Future Implications. Journal of Dental Research 2011 90(11):126470 .

2. Gerritsen AE, Allen PF, Witter DJ, Bronkhorst EM, Creugers NH. Tooth loss and oral health-related quality of life: a systematic review and metaanalysis. Health Qual Life Outcomes $20108(126$.

3. Bortoluzzi MC, Traebert J, Lasta R, Da Rosa TN, Capella DL, Presta AA. Tooth loss, chewing ability and quality of life. Contemporary Clinical Dentistry 2012 Oct-Dec;3(4):393-7.

4. Santillo PMH, Moura C, Coelho-Soares RS, Gusmão ES, Santos PCO. Impacto biopsicossocial da perda dentária em trabalhadores brasileiros de área rural. Pesqui prat psicossociais 2014 jul-dez;8(2):234-48.

5. Christensen GJ. Restoring a single anterior tooth: solutions to a dental dilemma. J Am Dent Assoc 2004 Dec;135(12):1725-7.

6. Gonzaga CC, Campos EA, BarattoFilho F. Restoration of endodontically treated teeth. RSBO 2011 JulSep;8(3):e33-46.

7. Gutmann JL. The dentin-root complex: anatomic and biologic considerations in restoring endodontically treated teeth. J Prosthet Dent 1992 Apr;67(4):458-67.

8. Teófilo LT, Zavanelli RA, Queiroz KV. Retentores intra-radiculares: revisão de literatura. PCL Rev Ibero-am prót clín laboratorial 2005 abr.-jun. ;7(36):18393.

9. Mota AS, Biffi JCG, Oliveira MRS, Guimarães CS. Estudo comparativo da força de tração na remoção de pinos pré-fabricados em canais morfologicamente diferentes. Rev ABO nac 2000 dez-jan;7(6):364-71.
10. Mazaro JVQ, Assunção WG, Rocha EP, Zuim PRJ, Genarri Filho H. Factors determining of intraradicular post selection. Rev odontol UNESP 2006 out-dez;35(4):223-31.

11. Conceição EN, Masotti A, Hirata R. Reproduzindo função e estética com compósitos diretos e indiretos em dentes posteriores. In: Conceição EN. Restaurações estéticas: compósitos, cerâmicas e implantes. Porto Alegre: Artmed; 2005.

12. Baratieri LN. Odontologia restauradora: fundamentos e técnicas. 2. ed. São Paulo: Santos; 2010.

13. Pereira RA, Franciscone PA, Porto CP. Cimentação de pinos estéticos com cimento resinoso: uma revisão. Rev Fac Odontol 2005 17(1):43-7.

14. Souza Júnior JA, Santos PH. Pinos pré-fabricados e sua cimentação: artigo de revisão. Rev Odontol Bras Central 2002 11(32):42-5.

15. Shiozawa LJ, Capp Cl, Mandetta S, Cara AA, Tamaki R. Retenção de pinos pré-fabricados e núcleos metálicos fundidos cimentados com cimento resinoso e fosfato de zinco. RPG rev pos-grad 2005 abr-jun;12(2):248-54.

16. Santos Junior GC, Freitas AP, Bastos LGC, Pinheiro MF, Rivas CC. A evolução da resina composta. Rev Fac Odontol Univ Fed Bahia 2000 jan-jun;1(20):29-33.

17. Deliperi S. Direct fiber-reinforced composite restoration in an endodontically-treated molar: a three-year case report. Oper Dent 2008 MarApr;33(2):209-14.

18. Cheung W. A review of the management of endodontically treated teeth. Post, core and the final restoration. J Am Dent Assoc 2005 May; 136(5):6119.

19. Rossato DM, Saade EG, Saad JRC, Porto Neto ST. Coroas estéticas anteriores em cerâmica metal-free: relato de caso clínico. RSBO 2010 7(4):494-8.
DE BITENCOURT $P V M$

GANDOLFI SAN

SILVA HA

DAMO DM

AROSSI GA

COROA TOTAL EM RESINA COMPOSTA

DIRETA: RELATO

de dOIS casos

CLÍNICOS
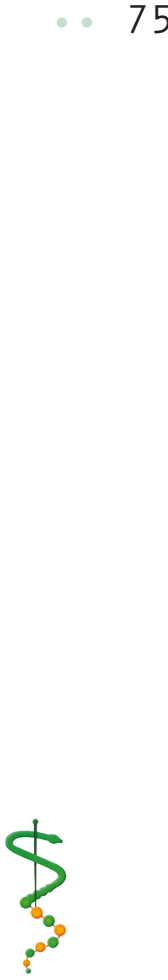

REV, ODONTOL.

UNIV, CID, SÃO

PAULO

$2016 ; 28(1): 65$

77 , JAN-ABR 


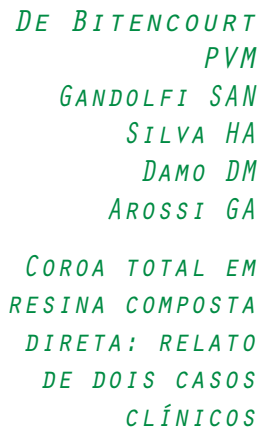

GANDOLFI SAN

SILVA HA

DAMO DM

AROSSI GA

COROA TOTAL EM

RESINA COMPOSTA

DIRETA: RELATO

DE DOIS CASOS

CLÍNICOS

76

\section{SSN 1983-5183}

20. Arossi GA, Reichert LA, Busato ALS. Coroas totais de resina composta direta: relato de casos clínicos. Rev Fac Odontol Porto Alegre 2010 maio-ago;51(2):31-8.

21. Muniz L, Góes CF, Oliveira ACPC, Mathias $\mathrm{P}$, Bezerra RB, Fontes CM. Restaurações diretas associadas a pinos de fibra de vidro em dentes fraturados. Relato de caso clínico. Rev Dental Press Estét 2005 jul/ago/set;2(3):45-57.

22. Turkun LS, Turkun M. The effect of one-step polishing system on the surface roughness of three esthetic resin composite materials. Oper Dent 2004 Mar-Apr;29(2):203-11.

23. Hirata R, Ampessan RL, Liu J. Reconstrução de dentes anteriores com resinas compostas - uma sequência de escolha e aplicação de resinas. $J B C$, J Bras Clin Estet Odontol 2001 jan-fev; 5(25):15-25.

24. Manfio AP, Biacchi GR, Guimarães MB, Habekoste FM. Coroa total de resina composta: procedimento alternativo na reconstrução do elemento dental. RGO, Porto Alegre 2006 jan-mar;54(1):27-30.

25. Moro M, Agostinho AM, Matsumoto W. Núcleos metálicos fundidos $\mathrm{x}$ pinos pré-fabricados. PCL Rev Ibero-am prót clín laboratorial 2005 abr.-jun. ;7(36):167-72.

26. Amaral M. Condicionamento da superfície de pinos de fibra de vidro: influência na resistência adesiva após ciclagem mecânica e na resistência à flexão dos pinos [Dissertação]. Santa Maria, RS: Universidade Federal de Santa Maria; 2010. $61 \mathrm{f}$.

27. Malferrari S, Monaco C, Scotti R. Clinical evaluation of teeth restored with quartz fiber-reinforced epoxy resin posts. Int J Prosthodont 2003 JanFeb;16(1):39-44.
28. Carvalho AMG, Renner SH, Almeida JV, Mondelli RFL, Valera RC. Análise crítica dos pinos intraradiculares de cerâmica, fibra de carbono e fibra de vidro. JBD Rev Ibero-am odontol estét dentística 2005 jan.-mar.;4(13):37-46.

29. Marturelli R, Cavalcanti NM, Souza FB, Porto POB, Silva CH. Alternativa estética para reconstrução de dentes anteriores fraturados. Stomatos 2007 jul-dez;13(25):123-30.

30. Menezes MS. Influência do cimento endodôntico na adesão do pino de fibra de vidro à dentina radicular [Dissertação]. Uberlândia, MG: Universidade federal de Uberlândia; 2006. 54 f.

31. Silva RVC, Veronezi MC, Dekon AFC, Silva PMB, Silva LM, Andrade AM. Comparação da resistência à tração entre pinos metálicos $(\mathrm{Ni} / \mathrm{Cr})$ e de fibra de virdro cimentados com cimento resinoso. Salusvita 2009 28(1):41-51.

32. Korkut B, Yanıkoğlu F, Günday M. Direct Composite Laminate Veneers: Three Case Reports. Journal of Dental Research, Dental Clinics, Dental Prospects 2013 Spring;7(2):105-11.

33. Gomes GM, Arana LA, Gomes OMM, Loguercio AD, Reis A, Calixto AL. Influência do operador e sistema de cimentação na retenção de pinos de fibra. IX Encontro de Pesquisa e III Simpósio de Pós-Graduação: Ponta Grossa/PR; 2010.

34. Andrade CL, Goncalves TM, Santos IL, Barros MS, Araujo NR, Cury AA. Direct adhesive pin-retained restorations for severely worn dentition treatment: a 1.5-year follow-up report. Braz Dent J 2014 25(4):357-62.

35. Paolone G, Saracinelli M, Devoto W, Putignano A. Esthetic direct restorations in endodontically treated anterior teeth. Eur J Esthet Dent 2013 Spring;8(1):44-67. 
36. Souza FHC, Vendrusculo AM, Barcelos CF, Lopes GS, Onófio GL. Reconstrução coronária com resina composta e pino estético intracanal. J Clin Dent 2002 1(15):52-7.

37. Demarco FF, Correa MB, Cenci MS, Moraes RR, Opdam NJ. Longevity of posterior composite restorations: not only a matter of materials. Dent Mater 2012 Jan;28(1):87-101.
38. Wolff D, Kraus T, Schach C, Pritsch M, Mente J, Staehle HJ, et al. Recontouring teeth and closing diastemas with direct composite buildups: a clinical evaluation of survival and quality parameters. J Dent 2010 Dec;38(12):1001-9.

Recebido em 14/04/2016

Aceito em 08/08/2016

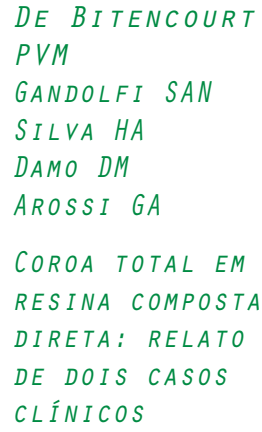

